\title{
Schizophrenia and frontotemporal dementia: Shared causation?
}

\author{
MICHAE HARCIAREK ${ }^{1}$, DOLORES MALASPINA ${ }^{2}$, TAO SUN ${ }^{3}$ \& \\ ELKHONON GOLDBERG ${ }^{4}$
}

\author{
${ }^{1}$ Division of Clinical Psychology and Neuropsychology, Institute of Psychology, University of Gdansk, Poland, \\ ${ }^{2}$ Department of Psychiatry, New York University School of Medicine, New York, USA, ${ }^{3}$ Department of Cell \\ and Developmental Biology, Cornell University Weill Medical College, New York, USA, ${ }^{4}$ Department of Neurology, \\ New York University School of Medicine, New York, USA
}

\begin{abstract}
The relationship between specific genes and particular diseases in neuropsychiatry is unclear, and newer studies focus on shared domains of neurobiological and cognitive pathology across different disorders. This paper reviews the evidence for an association between schizophrenia and frontotemporal dementia, including symptom similarity, familial co-morbidity, and neuroanatomical changes. Genetic as well as epigenetic findings from both schizophrenia and frontotemporal dementia are also discussed. As a result, we introduce the hypothesis of a shared susceptibility for certain subgroups of schizophrenia and frontotemporal dementia. This common causation may involve the same gene(s) at different stages of life: early in schizophrenia and late in frontotemporal dementia. Additionally, we provide a rationale for future research that should emphasize both genetic and cognitive parallels between certain forms of schizophrenia and frontotemporal dementia in a synergistic, coordinated way, placing both in the context of aberrant lateralization patterns.
\end{abstract}

\section{Introduction}

The relationship between specific genes and particular diseases in neuropsychiatry remains unclear, and the understanding of this relationship continues to evolve, reflecting the changes in the neuroscientific zeitgeist. Variable expressivity, pleiotrophic effects, partial penetrance, epistasis, and gene-environment interactions have been proposed to explain different disease presentations related to the same genes over time and between affected individuals. This relationship is made particularly elusive when cognitive and affective symptoms are used to construct the diagnostic entities, since the nature of such symptoms is determined primarily by the neuroanatomy that is involved, and only secondarily by pathophysiology. Also, the same neuroanatomical structure is under control of numerous other regions and genes, many or most of which may change in expression over development and as a consequence of life exposures. This absence of one-to-one (isomorphic), or even of many-to-one (homomorphic) relationship between genes and disorders has made the investigation of any relationship between them particularly daunting.

The failure to find major 'culprit' genes behind many neuropsychiatric conditions has forced a re-examination of what constitutes a 'disorder' in neuropsychiatry, and invited the revision of, and departure from, traditional diagnostic taxonomies. The notion that domains of psychopathology reflect a graduated symptom space is increasingly embraced to replace the traditional notions of 'disorders' that are separated by inherently discrete boundaries. This paradigm shift has made it conceptually feasible to draw parallels between disorders that were traditionally regarded as taxonomically distant and unrelated. Thus, schizophrenias and dementias would seem to be particularly distant taxonomically - the former have been recently envisioned as neurodevelopmental disorders characterized by psychotic symptoms, whereas the others are disorders of cognition and affect as a consequence of pathological ageing. Yet similar degenerative factors may be at play behind these conditions. Emil Kraepelin (1919) first recognized the similarity of these disorders, describing schizophrenia as a dementia that emerges in young adults (dementia praecox). Subsequent nosologies focused on other symptoms that Kraepelin considered to be only secondary to the neurodegenerative process. These illness features include psychosis, which entails delusions and hallucinations, disordered

Correspondence: Elkhonon Goldberg, PhD, ABPP-CN, NYU School of Medicine, 315 West 57th Street, Suite 401, New York, NY 10019 , USA. Tel + 1212 541 6412. Fax + 1212246 8916. E-mail: elkhonon.goldberg@nyumc.org 
thinking and bizarre behaviour (defined as 'positive' symptoms), and deficits in emotional expression and volition (defined as 'negative' symptoms).

Frontotemporal dementia (FTD) is a particularly good example of a neurodegenerative disorder characterized by shared properties and perhaps even shared underlying causal factors with schizophrenias. FTD is a midlife onset clinical condition first described by Arnold Pick (1892) and previously known as Pick's disease (Kertesz, 2008). It is characterized by progressive behavioural and personality abnormalities and/or language impairment that have been associated with the degeneration of frontal and/ or temporal regions of the brain (Harciarek \& Jodzio, 2005). The clinical presentation of FTD is heterogeneous, and FTD is commonly divided into two subtypes. The first subtype of FTD is its behavioural variant, with impaired social conduct, disinhibition, stereotypic behaviours, loss of empathy, as well as apathy among its most prominent features (Raskovsky et al., 2011). The second subtype of FTD is its language variant, also often described as primary progressive aphasia (PPA) (Hodges, 2007; Mesulam, 2003). According to the most recent classification, PPA can be further divided into three variants: semantic, non-fluent/agrammatic, and logopenic (Gorno-Tempini et al., 2011; Harciarek \& Kertesz, 2011; Mesulam et al., 2008). Of note, in comparison to the semantic and the non-fluent/agrammatic PPA predominantly associated with frontotemporal lobar degeneration (FTLD), the logopenic variant has been more often linked to the pathology of Alzheimer's disease (Grossman, 2010; Mesulam et al., 2008; Rabinovici et al., 2008).

Although schizophrenia and FTD have been typically described in terms of specific feature constellations characteristic for each disorder (e.g. younger onset and more pronounced delusions as well as hallucinations in schizophrenia), it has been also shown that there is a great deal of clinical, neuroimaging, genetic, and pathological overlap, making it sometimes difficult to distinguish between these two conditions (Momeni et al., 2010a). The association between schizophrenia and FTD has been rarely and only recently studied, however, despite Kraepelin's early observations. This may, at least in part, reflect the diagnostic criteria applied for schizophrenia and FTD, as well as the traditional dominance of rigid diagnostic taxonomies. For example, subjects with a history of neurological conditions are typically not included in studies of schizophrenia. Also, widely used diagnostic criteria for FTD (McKhann et al., 2001; Neary et al., 1998) do not encompass assessment of psychotic symptoms, although these have been known to appear in FTD (Chow et al., 1999; Le Ber et al., 2006, 2008; Mendez et al., 2008; Schoder et al., 2010).
Difficulties with differentiating between schizophrenia-related psychosis and neurodegenerative disorders suggestive of certain common underlying factors are not new, and they predate the original work by Kraepelin (1919) and Pick (1892). One such ambiguous case was the case of Ludwig II, King of Bavaria (1845-1886), whose medical history has been recently reviewed by Forstl et al. (2008). According to the royal medical report, significant and rather progressive changes in the personality and behaviour of Ludwig II were noted when he reached the age of about 25 . He became withdrawn and, as with many patients with FTD became obsessed with irrelevant details. His speech became odd (although its specific characteristics were not recorded) and he became aggressive. $\mathrm{He}$ also became delusional with frequent hallucinations, this bringing his clinical picture towards schizophrenia-like psychosis. Interestingly, however, he seemed to be well aware of his abnormal behaviour but did not attempt to alter it, despite the apparent insight. With age, certain motor symptoms developed, which are common in both schizophrenia and FTD. According to Forstl et al. (2008), the description of Ludwig's symptoms is congruent with the diagnosis of schizophrenia disorder according to DMS-IV. This diagnosis is further supported by a positive family history for schizophrenia-like psychosis; both Ludwig's younger brother Otto as well as the youngest daughter of Ludwig's grandfather developed severe mental disorder with inappropriate affect, catatonic behaviour and apathy from an early age until death. At the same time, a post-mortem examination of Ludwig II, who died in unclear circumstances (possibly drowning), revealed extensive frontal atrophy in both hemispheres suggestive of a neurodegenerative disease.

In this paper we review the evidence for a relationship between schizophrenia and FTD and introduce the hypothesis that their respective causations could be manifestations of the abnormalities involving the same genes at different life stages: early in schizophrenia and late in FTD. Of course, both schizophrenia and FTD are syndromes consisting of heterogeneous components, so any hypothesis pertaining to the similarity of underlying mechanisms is likely to be applicable only to certain subtypes of schizophrenia and subtypes of FTD. We will examine several sources of evidence pertaining to the relationship between schizophrenia and FTD: (1) symptom similarity, (2) familial co-morbidity, (3) neuroanatomy, (4) genetics, and (5) epigenetics.

\section{Shared symptomatology in schizophrenia and FTD}

The concept of negative and positive symptoms was first introduced by John Russell Reynolds (1858) to 
illuminate the features of epilepsy. In his view, negative symptoms were the loss of vital properties that could cause conditions such as paralysis or anaesthesia, while positive symptoms were the excess of vital properties resulting in conditions such as spasms, pain or convulsions. Although both could be observed at the same time, Reynolds believed that positive and negative symptoms were independent of each other (Berrios, 1985). Expanding on Reynolds idea, Hughlings Jackson (1958) conceptualized positive symptoms as the result of the loss of higher inhibitory mechanisms that permitted excitation or the discharge of lower neural systems and negative symptoms as the result of reduced neural function. Unlike Reynolds, Jackson believed that positive and negative symptoms were different manifestations of a single process. Kraepelin (1919) and Bleuler (1908) adopted Jackson's theory of positive and negative symptoms in their conceptualizations of schizophrenia. A shared aetiopathophysiology for cases of schizophrenia and FTD would be most consistent with Jackson's model. Deterioration of frontal lobes with secondary release of positive symptoms would fit this view.

As already suggested, similarity of symptomatology of schizophrenia and FTD and the potential for diagnostic confusion between them has been noted by several authors (Duggal \& Singh, 2009; Vanderzeypen et al., 2003; Velakoulis et al., 2009; Waddington et al., 1995). This seems to be a serious clinical problem, especially since both the therapeutic interventions and prognosis of these disorders are quite different.

Schizophrenia is normally characterized by the onset of so-called positive symptoms (e.g. delusions, hallucinations) during adolescence or young adulthood. Nonetheless, similarly to FTD, the clinical presentation of schizophrenia is heterogeneous, encompassing also a variety of negative symptoms, such as lack of volition, personal neglect, social withdrawal, and such cognitive symptoms as executive deficit and/or language impairment. The neuropsychological profile identified in schizophrenic patients is usually characterized by executive deficits (Berman et al., 1986), although they are often less severe than seen in FTD. Although dementia is not typically considered to occur in schizophrenia, nor is it clearly recognized as an obligatory complication of this disorder, many subjects with schizophrenia develop disorientation and cognitive impairment (de Vries et al., 2001). Furthermore, later-life cognitive deterioration in a subset of schizophrenic patients is well described (Harvey, 2012); such schizophrenia cases have sometimes been called 'Kraepelinian subtypes' in the research literature.

The exact mechanism or mechanisms underlying positive and negative symptoms in schizophrenia remains an open question. It is possible that whereas positive symptoms are related to an increase in dopamine in the mesolimbic pathway, a defective dopamine function in the mesocortical pathway may contribute to the development of negative symptoms (Weinberger \& Berman, 1988). Further, it has been proposed that negative symptoms represent a consequence of impaired frontal lobe function (Bergé et al., 2011; Frith, 1992; Liddle, 1987; Weinberger, 1988). In fact, when Kraepelin (1919) introduced the concept of 'dementia praecox', he suggested that, based on the symptomatology of schizophrenia, it should be considered a frontotemporal disorder. Also, many authors have noted that the affective flattening, lack of volition, poor judgement, personal neglect and social withdrawal seen in many patients with schizophrenia are among symptoms typically seen in subjects with disease of the frontal lobe (Frith, 1992; Levin, 1984; Liddle, 1987; Pantelis et al., 1992; Weinberger, 1988; Winograd-Gurvich et al., 2006). Additionally, it has been shown that such negative symptoms are related to defective performance on neuropsychological tests sensitive to frontal lobe function, on tests of attention and executive abilities in particular (Dibben et al., 2009; Donohoe et al., 2006).

Although clinical signs of schizophrenia are similar to those seen in frontal dysfunction, and thus, may overlap with symptoms of FTD, only few recent studies directly compared the symptomatology of schizophrenia and FTD (Kosmidis et al., 2008; Weickert et al., 2011; Velakoulis et al., 2009; Ziauddeen et al., 2011). Ziauddeen et al. (2011) compared a small group of negative-symptom schizophrenic patients and patients with the behavioural variant FTD and found that both groups displayed negative symptoms typically seen after frontal lobe injury. Specifically, high rate of apathy, loss of empathy and stereotypic behaviours were noted in both conditions. Also, patients with schizophrenia presented with a relatively similar neuropsychological profile to those with FTD on neuropsychological tests, both groups particularly impaired on tests of executive functions, although this impairment was greater in FTD. Further, speech abnormalities were seen in both disorders, although they were more common in patients with schizophrenia (the latter finding being inconclusive, since subjects with the language variant FTD were not included in the study). Kosmidis et al. (2008) compared patients with schizophrenia with those suffering from FTD and found impaired performance on measures of theory of mind in both groups. Weickert et al. (2011) showed that impaired probabilistic association learning is equally impaired in schizophrenia and in FTD (particularly in the behavioural variant). 
Whereas numerous studies have provided good evidence for a significant overlap in negative symptoms between schizophrenia and FTD, positive symptoms in FTD have been relatively rarely studied. In the few reported studies only a small subset of patients with FTD (up to $13.3 \%$ ) were found to have psychotic episodes either at onset or during the course of illness (Ibach et al., 2004; Lindau et al., 2000; Mendez et al., 2008; Piguet et al., 2004; Seeley et al., 2005; Velakoulis et al., 2009). The occurrence of psychosis in FTD seems to be significantly higher in subjects with relatives suffering from schizophrenia or schizoaffective disorder (Schoder et al., 2010). Thus, although the percentage of positive symptoms in FTD seems to be rather small, psychotic symptoms do appear in this particular neurodegenerative condition, even in subjects with causal FTD mutation (Momeni et al., 2010a, 2010b; Schoder et al., 2010; Snowden et al., 2012). This implies that their presence should not necessarily exclude the diagnosis of FTD (Duggal \& Singh, 2009).

The clear overlap between negative symptoms in psychiatry and many behaviour abnormalities seen after frontal lobe injury (e.g. emotional indifference) or dementias suggests that they may represent the same clinical phenomenon (Kleist, 1960). Hence, lack of volition in schizophrenia could be the same phenomenon as apathy in FTD, as posited by Ziauddeen et al. (2011). Similarly, the poverty of speech and its content in schizophrenia could mirror logopenia, semantic and pragmatic language disturbances, as well as speech perseverations often seen in patients with FTD. Indeed, perseveration and field-dependent behaviour (i.e. behaviour driven by incidental task-irrelevant external or internal stimuli), commonly seen after frontal-lobe damage, accounts for much of the language disorder in schizophrenia (Barr et al., 1989). Likewise, motor perseveration characteristic of patients with frontal-lobe damage (Goldberg \& Tucker, 1979) is also observed in schizophrenia, and they may be practically indistinguishable in the two populations (Bilder \& Goldberg, 1987). Also, behaviours associated with delusional beliefs (e.g. repeated phone calls to the police) may, to some extent, overlap with abnormal behaviour of patients with FTD and, thus, influence the diagnosis (Snowden et al., 2012). Future systematic studies are needed in order to better understand the relationship between the cognitive symptoms in schizophrenia and FTD.

\section{Familial and individual co-morbidity in schizophrenia and FTD}

To date, only a few studies have addressed the question of familial co-morbidity in schizophrenia and FTD. Schoder et al. (2010) compared morbid risk for schizophrenia and schizoaffective disorder in the first-degree relatives of 100 FTD probands to that in a group of first-degree relatives of 100 Alzheimer's disease $(\mathrm{AD})$ probands. They noted that schizophrenia-related psychosis was seen significantly more often in relatives of the FTD than AD probands. Also, the occurrence of psychosis in FTD was significantly higher in subjects with relatives suffering from schizophrenia or schizoaffective disorder (Chow et al., 1999).

Additionally, 10 mixed families (with both FTD and schizophrenic family members) were identified, and in three of them an FTD causal mutation was determined (Schoder et al., 2010). This mutation was also present in the schizophrenic family members, suggesting a common aetiology for both disorders in some families. In this particular cohort schizophrenia was diagnosed in young individuals (before age 40), the diagnosis remained stable for approximately 17 years, and all of the participants diagnosed with schizophrenia experienced positive symptoms rarely seen in FTD (Mendez et al., 2008). Moreover, the psychotic symptoms in patients with schizophrenia were effectively treated with antipsychotic medication, whereas subjects with FTD usually do not benefit from such treatment, have more motor side effects and may even experience an earlier death with these treatments (Kerchner et al., 2011). Thus, the possibility of misdiagnosis of FTD for schizophrenia in the study by Schoder et al. (2010) seems unlikely. High familial co-morbidity of schizophrenia and FTD has been shown also by Momeni et al. (2010a, 2010b).

Nonetheless, the clinical overlap between schizophrenia and FTD may sometimes contribute to misdiagnosing FTD as schizophrenia, particularly in the less common cases of early onset FTD (Momeni et al., 2010a; Velakoulis et al., 2009), or in cases where late onset schizophrenia is a differential diagnosis (Lagodka \& Robert, 2009; Reischle et al., 2003). For example, Momeni et al. (2010a) described two members of the same family diagnosed with FTD and Parkinsonism linked to chromosome 17 (FTDP-17), here due to a novel exon 12 mutation in tau pathology. The review of medical records of these individuals revealed that both the proband and her father were initially diagnosed with schizophrenia.

A possibility also exists that an early-adulthood schizophrenic syndrome may represent a genuine prodromal phase of FTD indicating an individual co-morbidity. This hypothesis is supported by the study by Waddington et al. (1995), who reported a woman initially diagnosed with paranoid schizophrenia due to auditory hallucinations and persecutory delusions. Nonetheless, a few years after the onset of psychosis, her clinical picture changed and she developed affective abnormalities as well as cognitive 
deficits suggestive of FTD. Neuroimaging revealed frontotemporal atrophy and brain biopsy revealed neuropathological evidence of Pick's disease; i.e. numerous neurons were found to contain spherical intracytoplasmic inclusion tau-positive bodies. Also, immunohistochemistry for glial fibrillary acidic protein revealed dense pancortical astrogliosis with additional subcortical gliosis. Based on these observations, Waddington et al. concluded that although it could not be ruled out that the patient was unfortunate enough to develop two independent disease processes, it was more likely that 'some specific aspect of the pathology of Pick's disease in this patient has, early in its course, disrupted brain function in a manner that reproduces some fundamental aspects of the pathophysiology of schizophrenia.' Similar cases of early-adulthood schizophrenic syndrome prodromal to FTD have been reported (Kitabayashi et al., 2005; Vanderzeypen et al., 2003). Velakoulis et al. (2009) described four patients with FTD who at a young age developed psychotic symptoms and received the diagnosis of schizophrenia. In contrast to the reports of initial schizophrenia-like psychosis with pathologically confirmed FTDtauopathy (Waddington et al., 1995), the above cases reported by Velakoulis et al. exhibited pathological changes characteristic of FTD with motor neuron disease (FTD-MND) (i.e. TAR DNA-binding protein 43 neuronal inclusions; TDP-43 positive). The association between FTD, TDP-43, and psychosis has also been suggested by Snowden et al. (2012). Still, not enough is known about the neuropathology of FTD cases initially presenting as psychotic disorder, and future studies are necessary.

Just as the true cases of FTD may be mistaken for schizophrenia, the opposite may also occur. Namely, in some individuals a clinical picture mirroring that of FTD may in fact be due to a later onset psychiatric disorder. Nonetheless, recent studies have shown that such cases, often referred to as 'FTD-phenocopy' (Hornberger et al., 2008, 2009, 2010; Irish et al., 2011), rarely present with cognitive impairment. Furthermore, their behavioural changes do not typically progress, and these patients do not show the characteristic frontotemporal atrophy on neuroimaging (Davies et al., 2006; Kipps et al., 2007; Pennington et al., 2011).

\section{Neuroanatomy of schizophrenia and FTD}

Cumulative evidence implicates similar structures in schizophrenia and FTD, namely the prefrontal cortex and anterior temporal lobes (Brewer et al., 2005; Buckley et al., 1999; Hill et al., 2004; Lawrie \& Abukmeil, 1998; Raskovsky et al., 2011; Shenton et al., 2001; de Vries et al., 2000; Waddington, 1995).
In fact, neuropsychological, neuroimaging and pathological studies have provided extensive support for the notion that in both schizophrenia and FTD negative symptoms as well as other critical behavioural and cognitive changes (e.g. perseverations) are related to abnormalities in the prefrontal cortex (Bonilha et al., 2008; Frith, 1992; Liddle, 1987; Weinberger, 1988). By contrast, psychotic symptoms (e.g. auditory hallucinations) may be predominantly related to pathology in the temporal cortex (Sumich et al., 2002), although in a recent study by Snowden et al. (2012) no differences in terms of frontal or temporal atrophy as well as hemispheric asymmetries were found between FTD patients with and without psychosis. Furthermore, specific subdivisions within the frontal and the temporal cortex are often implicated both in schizophrenia and in FTD - orbital subdivisions of the prefrontal cortex and anterior subdivisions of the temporal lobe (Hodges, 2007; Seeley et al., 2005; Shenton et al., 2001). In both disorders, functional and structural pathology may additionally encompass the thalamus and the basal ganglia (Rosenberg, 1983). Finally, the involvement of these structures appears to be lateralized in both disorders; they are more impacted in the left than in the right hemisphere both in schizophrenia (Bergé et al., 2011; Chance et al., 2008; Honer et al., 1996; Luauté et al., 1994; Puri et al., 1994; Schobel et al., 2009; Spironelli et al., 2011; van den Heuvel et al., 2010; van Haren et al., 2011; Waddington et al., 1995; Wolf et al., 2008) and in FTD (Boccardi et al., 2003; Jeong et al., 2005; Kanda et al., 2008; Rohrer et al., 2012; Whitwell et al., 2005, 2012).

Of note, orbitofrontal and anterior temporal regions are characterized by shared morphometric properties also in the normal brain: both are larger in the left than in the right hemisphere (Goldberg et al., 2013; Luders et al., 2006). The fact that these two structures are characterized by the same direction of lateralization both in the healthy brain and in the two disorders of interest raises the question of certain shared factors (generic, epigenetic, or others) controlling certain aspects of their development. Is it possible, for instance, that these disorders are caused by an aberrant expression of the same gene(s) but at different stages of life: early in schizophrenia and late in FTD?

\section{Aetiology and pathogenesis in FTD and psychosis}

Some studies have shown that $30-50 \%$ of FTD is familial, suggesting a genetic root for FTD (Seelaar et al., 2011; Ferrari et al., 2011). Mutations of progranulin $(G R N)$ and microtubule associated protein tau $(M A P T)$ account for about $50 \%$ of the familial 
FTD cases. GRN is involved in cell cycle progression, growth control, wound healing and in many other biological processes. It also displays abundant expression in the brain. By comparison, MAPT binds and stabilizes microtubules and plays an important role in neuronal polarity and signal transduction. Hyperphosphorylation of tau may be also associated with schizophrenia, and it has been suggested that dysregulation of reelin contributes to hyperphosphorylation of tau in schizophrenia (Deutsch et al., 2006). Thus, tau pathology may be associated with both FTD and schizophrenia, and reelin may be one of the regulators of tau function. Future studies are needed, however, to test these hypotheses.

Magnetic resonance imaging studies have shown strikingly asymmetric atrophy in brains of FTD patients, particularly in the GRN carriers (Beck et al., 2008; Rohrer et al., 2011; Whitwell et al., 2012). This asymmetry is often detected in the frontal, temporal and parietal regions. Moreover, the rate of atrophy in the left hemisphere is higher than that in the right (Rohrer et al., 2012). However, FTD patients carrying $M A P T$ mutations normally don't show asymmetric atrophy (Rohrer \& Warren, 2011). These studies suggest an association of specific FTD gene mutations with asymmetric atrophy during disease progression.

Intriguing questions then arise, whether genes mutated in FTD and/or schizophrenia are asymmetrically expressed in the normal human brain, and whether their mutations are associated with abnormal brain laterality and pathogenesis of FTD and schizophrenia. Previous studies have shown that certain genes are asymmetrically expressed in human foetal brains (Sun et al., 2005, Sun \& Walsh, 2006). Among these genes, MAPT has higher expression levels in the left than in the right hemisphere (more than two-fold) in human foetal 14-week-old brains (Sun et al., 2005). In human embryonic 10-week-old brains Reelin is also more highly expressed in the left than in the right hemisphere (about 1.6-fold) ( $\mathrm{T}$. Sun, unpublished data). Although a larger sample size is required to draw a comprehensive gene expression profile, these studies suggest that at least Reelin and $M A P T$ may participate in the process of establishing normal brain laterality. Mutations of Reelin and MAPT may disrupt normal asymmetry and contribute to the pathology of FTD and schizophrenia. It is yet to be understood why FTD patients with $M A P T$ mutations mostly show symmetric brain atrophy patterns, whereas patients with $G R N$ mutations show asymmetric atrophy in the brain. Nevertheless, investigating the molecular regulation of brain asymmetry will likely provide insights into the aetiology of FTD and schizophrenia.

Interestingly, Snowden et al. (2012) have recently noted that psychosis in patients with FTD is strongly associated with a hexanucleotide repeat expansion in C9ORF72 gene, a mutation found in approximately $6 \%$ of sporadic, and $25 \%$ of familial cases with amyotrophic lateral sclerosis (ALS; a very common form of MND) and FTD (Rademakers, 2012). In fact, the presence of psychosis increased the odds of having the C9ORF72 gene mutation by 15 -fold. Thus, it is possible that mutation in the C9ORF72 gene may be a major cause not only of FTD and MND (DeJesusHernandez et al., 2011; Renton et al., 2011), but also of late onset psychosis (Ferrari et al., 2012; Murray et al., 2011). Of note, aside from FTD-MND, a relatively high prevalence of psychosis has been noted in rare cases with fused-in-sarcoma (FUS), a pathology also associated with MND (Seelaar et al., 2010; Snowden et al., 2011). However, despite the above, there is a phenotypic variability in regard to the C9ORF72 repeat expansions. For example, cases with behavioural variant FTD without MND as well as PPAs without psychosis have been documented (Renton et al., 2011; Snowden et al., 2012). The neuropathology may be also heterogeneous (Murray et al., 2011; Snowden, 2012). Further, in a recent study, Huey et al. (2012) screened DNA samples from a large cohort of unrelated subjects with schizophrenia for mutation in the C9ORF72 gene. Interestingly, none of these subjects had the C9ORF72 repeat expansions, suggesting this mutation is unlikely to cause schizophrenia. Thus, caution needs to be exercised when determining the association between mutation in C9ORF72 gene, FTD phenotype, the presence of psychosis, and schizophrenia.

\section{Shared causation and future research directions}

The possibility of a shared causation between schizophrenia and certain dementias has been raised before (DeLisi, 2008; Schoder, 2010). The present review provides additional rationale for pursuing this line of research further. Such future research will emphasize both genetic and cognitive parallels between certain forms of schizophrenia and FTD in a synergistic, coordinated way. It is also increasingly possible to envision genetic research provide incrementally precise answers to the following questions:

1. Are the genes particularly strongly expressed in the two regions most commonly implicated in FTD and schizophrenia - orbitofrontal cortex and anterior temporal regions - characterized by asymmetric expression?

2. Are some of such asymmetrically expressed genes the same in the orbitofrontal cortex and the anterior temporal regions?

3. Are some of these genes implicated both in schizophrenia and in FTD? 
Inquiry into shared genetic mechanisms of diverse diseases should be conducted in parallel with the inquiry into the cognitive ramifications of altered gene expression. Advances in research methodology make it increasingly possible to manipulate gene expression in living animal models and to study the behavioural and cognitive consequences of such manipulations. In particular, it is increasingly possible to manipulate the degrees and direction of their asymmetric expression in the two hemispheres.

At the same time, the conceptual understanding of hemispheric specialization has advanced in ways that make parallels between animal models and human cognition (both normal and clinically aberrant) more feasible. The traditional understanding of hemispheric specialization emphasized the complementary roles of the two cerebral hemispheres in language versus visuo-spatial functions. Clearly, such conceptual framework has no meaning outside the human species, and thus precludes any parallels between animal and human cognition.

More recently, very different approaches to hemispheric specialization have been gaining prominence. One of them emphasizes the differential roles of the two hemispheres in novelty seeking (the right hemisphere) versus exercise of established routinized behaviours (the left hemisphere) (Goldberg \& Costa, 1981; Goldberg \& Podell, 1995; Goldberg et al., 1994b). Within this general framework, the lateralized nature of the effects of frontal-lobe damage in humans has been demonstrated. Damage to the left prefrontal regions results in an extremely field-independent (rigid perseverative) response selection, whereas damage to the right prefrontal regions results in an extremely field-dependent (unstable, excessively variable) response selection in humans (Goldberg \& Podell, 1995; Goldberg et al., 1994a, 1994b). Furthermore, sex differences in the consequences of lateralized prefrontal damage in humans have been described (Goldberg et al., 1994a, 1994b).

Unlike the classic language-visuospatial distinction, the contrasts between novelty-seeking and routinized stereotypical behaviours and between perseverative and field-dependent behaviours are meaningful across species and can be examined in animal models. In humans, these dimensions of cognition are critical both in characterizing individual differences between normal cognitive styles, and in characterizing cognitive pathology. Perseverative rigidity of mental processes on the one hand, and their tangentiality, looseness and field-dependency on the other, have been noted in describing both schizophrenic cognition and that of patients with various forms of dementia.

Thus, it may be possible to ascertain the cognitive effects of cortical gene manipulation in the two hemispheres in animal models using constructs that allow direct parallels with human cognition and with human clinical pathology. Experimental cognitive paradigms that permit the assessment of noveltyseeking versus propensity toward routinized behaviours, perseveration versus field dependency in parallel human and animal experiments already exist, and new experiments can be readily designed. The same may be true for other cognitive constructs meaningful across species and relevant to characterizing human psychopathology. The use of such paradigms will permit the drawing of parallels between the cognitive effects of select gene manipulation in animal models on one hand, and clinical profiles in disorders such as certain forms of schizophrenia and FTD on the other hand. Such a research programme will hold the promise of advancing our understanding of the causation of major neuropsychiatric disorders, including the patterns of shared causation.

\section{Take-home points}

Discrete taxonomies of neuropsychiatric disorders may be outdated, and aberrant lateralization of gene expression may be an important causal factor in neuropsychiatric disorders.

\section{Acknowledgements}

We thank Dmitri Bougakov for his assistance with literature reviews in preparation of this paper.

Declaration of interest: While preparing this manuscript, M.H. was receiving a scholarship from the Polish Ministry of Science and Higher Education; and DM was supported by NIH RC1 MH088843 and K24 MH01699 (DM). The authors alone are responsible for the content and writing of the paper.

\section{References}

Barr, W., Bilder, R., Goldberg, E., Kaplan, E. \& Mukhergee, S. (1989). Neuropsychology of schizophrenic language. Fournal of Communication Disorders, 22, 327-349.

Beck, J., Rohrer, J.D., Campbell, T., Isaacs, A., Morrison, K.E., Goodall, E.F., ... Mead, S. (2008). A distinct clinical, neuropsychological and radiological phenotype is associated with progranulin gene mutations in a large UK series. Brain, 131, 706-720.

-Bergé, D., Carmona, S., Rovira, M., Bulbena, A., Salgado, P. \& Vilarroya, O. (2011). Gray matter volume deficits and correlation with insight and negative symptoms in first-psychoticepisode subjects. Acta Psychiatrica Scandinavica, 123, 431-439.

Berman, K.F., Zec, R.F. \& Weinberger, D.R. (1986) Physiologic dysfunction of the dorsolateral prefrontal cortex in schizophrenia: II. Role of neuroleptic treatment, attention, and mental effort. Archives of General Psychiatry, 43, 126-135.

Berrios, E. (1985). Positive and negative symptoms and Jackson. A conceptual history. Archives of General Psychiatry, 42, 95-97. 
Bilder, R. \& Goldberg, E. (1987). Motor perseveration in schizophrenia. Archives of Clinical Neuropsychology, 2, 195-214.

Bleuler, E. (1908). Die Prognose der Dementia praecox [Prognosis for dementia praecox (schizophreniform type)]. Allgemeine Zeitschrift für Psychiatrie und Psychisch-Gerichtliche Medizin, 65, 436-464.

- Boccardi, M., Laakso, M.P., Bresciani, L., Galluzi, S., Geroldi, C., Beltramello, A., ... Frisoni, G.B. (2003). The MRI pattern of frontal and temporal brain atrophy in fronto-temporal dementia. Neurobiology and Aging, 24, 95-103.

Bonilha, L., Molnar, C., Horner, M.D., Anderson, B., Forster, L., George, M.S. \& Nahas, Z. (2008). Neurocognitive deficits and prefrontal cortical atrophy in patients with schizophrenia. Schizophrenia Research, 101, 142-151.

Brewer, W.J., Francey, S.M., Wood, S.J., Jackson, H.J., Pantelis, C., Phillips, L.J., ... McGorry, P.D. (2005). Memory impairments identified in people at ultra-high risk for psychosis who later develop first-episode psychosis. American fournal of Psychiatry, 162, 71-78.

Buckley, P.F., Dean, D., Bookstein, F.L., Friedman, L., Kwon, D., Lewin, J.S., ... Lys, C. (1999). Three-dimensional magnetic resonance-based morphometrics and ventricular dysmorphology in schizophrenia. Biological Psychiatry, 45, 62-67.

-Chance, S.A., Casanova, M.F., Switala, A.E. \& Crow, T.J. (2008). Auditory cortex asymmetry, altered minicolumn spacing and absence of ageing effects in schizophrenia. Brain, 131, 3178-3192.

-Chow, T.W., Miller, B.L., Hayashi, V.N. \& Geschwind, D.H. (1999). Inheritance of frontotemporal dementia. Archives of Neurology, 56, 817-822.

Davies, R.R., Kipps, C.M., Mitchell, J., Kril, J.J., Halliday, G.M. \& Hodges, J.R. (2006). Progression in frontotemporal dementia: Identifying a benign behavioral variant by magnetic resonance imaging. Archives of Neurology, 63, 1627-1631.

DeJesus-Hernandez, M., Mackenzie, I.R., Boeve, B.F., Boxer, A.L., Baker, M., Rutherford, N.J., ... Rademakers, R. (2011). Expanded GGGGCC hexanucleotide repeat in noncoding region of C9ORF72 causes chromosome 9p-linked FTD and ALS. Neuron, 72, 245-256.

DeLisi, L.E. (2008). The concept of progressive brain change in schizophrenia: Implications for understanding schizophrenia. Schizophrenia Bulletin, 34, 312-321.

de Vries, P.J., Honer, W.G., Kemp, P.M. \& McKenna, P.J. (2001). Dementia as a complication of schizophrenia. fournal of Neurology, Neurosurgery, and Psychiatry, 70, 588-596.

Deutsch, S.I., Rosse, R.B. \& Lakshman, R.M. (2006). Dysregulation of tau phosphorylation is a hypothesized point of convergence in the pathogenesis of Alzheimer's disease, frontotemporal dementia and schizophrenia with therapeutic implications. Progress in Neuropsychopharmacology and Biological Psychiatry, 30, 1369-1380.

Dibben, C.R., Rice, C., Laws, K. \& McKenna, P.J. (2009). Is executive impairment associated with schizophrenic syndromes? A meta-analysis. Psychological Medicine, 39, 381-392.

Donohoe, G., Corvin, A. \& Robertson, I.H. (2006). Evidence that specific executive functions predict symptom variance among schizophrenia patients with a predominantly negative symptom profile. Cognitive Neuropsychiatry, 11, 13-32.

Duggal, H.S. \& Singh, I. (2009). Frontotemporal dementia presenting with psychotic symptoms. Fournal of Neuropsychiatry and Clinical Neurosciences, 21, 103-104.

Ferrari, R., Hardy, J. \& Momeni, P. (2011). Frontotemporal dementia: From Mendelian genetics towards genome wide association studies. Fournal of Molecular Neuroscience, 45, 500-515.

Ferrari, R., Mok, K., Moreno, J.H., Cosentino, S., Goldman, J., Pietrini, P., ... Momeni, P. (2012). Screening for C9ORF72 repeat expansion in FTLD. Neurobiology of Aging, 33, 1850e1-1850.e11.

Frith, C.D. (1992). The Cognitive Neuropsychology of Schizophrenia. Hove: Erlbaum.
Förstl, H., Immler, G., Seitz, M. \& Hacker, R. (2008). Ludwig II, King of Bavaria: A royal medical history. Acta Psychiatrica Scandinavica, 118, 499-502.

- Goldberg, E. \& Costa, L. (1981). Hemisphere differences in the acquisition and use of descriptive systems. Brain and Language, 14, 144-173.

Goldberg, E. \& Podell, K. (1995). Lateralization in the frontal lobes. Advances in Neurology, 66, 85-96.

- Goldberg, E. \& Tucker, D. (1979). Motor perseverations and the levels of encoding a visual form. Fournal of Clinical Neuropsychology, 4, 273-288.

Goldberg, E., Podell, K. \& Lovell, M. (1994a). Lateralization of frontal lobe functions and cognitive novelty. Fournal of $\mathrm{Neu}$ ropsychiatry and Clinical Neurosciences, 6, 371-378.

- Goldberg, E., Podell, K., Harner, R., Lovell, M. \& Riggio, S. (1994b). Cognitive bias, functional cortical geometry, and the frontal lobes: Laterality, sex, and handedness. Fournal of Cognitive Neuroscience, 6, 274-294.

- Goldberg, E., Roediger, D., Kucukboyaci, N.E., Carlton, C., Devinsky, O., Kuzniecky, R., Halgren, E., Thesen, T. (2013). Hemispheric asymmetries of cortical volume in the human brain. Cortex, 49, 200-210.

Gorno-Tempini, M.L., Hillis, A.E., Weintraub, S., Kertesz, A., Mendez, M., Cappa, S.F., ... Grossman, M. (2011). Classification of primary progressive aphasia and its variants. Neurology, 76, 1006-1014.

Grossman, M. (2010). Primary progressive aphasia: Clinicopathological correlations. Nature Reviews Neurology, 6, 88-97.

Harciarek, M. \& Jodzio, K. (2005). Neuropsychological differences between frontotemporal dementia and Alzheimer's disease. Neuropsychology Review, 3, 131-145.

-Harciarek, M. \& Kertesz, A. (2011). Primary progressive aphasias and their contribution to the contemporary knowledge about the brain-language relationship. Neuropsychology Review, 21, 271-287.

Harvey, P.D. (2012). Cognitive impairment in schizophrenia: Profile, course, and neurobiological determinants. Handbook of Clinical Neurology, 106, 433-445.

Hill, K., Mann, L., Laws, K.R., Stephenson, C.M., Nimmo-Smith, I. \& McKenna, P.J. (2004). Hypofrontality in schizophrenia: A meta-analysis of functional imaging studies. Acta Psychiatrica Scandinavica, 110, 243-256.

Hodges, J.R. (2007). Frontotemporal Dementia Syndromes. New York: Cambridge University Press.

-Honer, W.G., Kopala, L.C., Locke, J.J. \& Lapointe, J.S. (1996). Left cerebral hemiatrophy and schizophrenia-like psychosis in an adolescent. Schizophrenia Research, 20, 231-234.

-Hornberger, M., Piguet, O., Graham, A.J., Nestor, P.J. \& Hodges, J.R. (2010). How preserved is episodic memory in behavioral variant frontotemporal dementia? Neurology, 74, 472-479.

Hornberger, M., Piguet, O., Kipps, C. \& Hodges, J.R. (2008). Executive function in progressive and nonprogressive behavioral variant frontotemporal dementia. Neurology, 7, 1481-1488.

Hornberger, M., Shelley, B.P., Kipps, C.M., Piguet, O. \& Hodges, J.R. (2009). Can progressive and non-progressive behavioural variant frontotemporal dementia be distinguished at presentation? fournal of Neurology, Neurosurgery, and Psychiatry, 80, 591-593.

Huey, E.D., Nagy, P.L., Rodriguez-Murillo, L., Manoochehri, M., Goldman, J., Lieberman, J., ... Mayeux, R. (2012). C9ORF72 repeat expansions not detected in a group of patients with schizophrenia. Neurobiology of Aging. doi: 10.1016/j. neurobiolaging.2012.08.011.

Hughlings Jackson, J. (1958). Selected Writings of fohn Hughlings fackson, J. Taylor, G. Holmes, \& F.M.R. Washie (Eds). London: Hodder \& Stoughton.

-Ibach, B., Poljansky, S., Barta, W., Koller, M., Wittmann, M. \& Hajak, G. (2004). Patterns of referring of patients with 
frontotemporal lobar degeneration to psychiatric in- and outpatient services. Results from a prospective multicentre study. Dementia and Geriatric Cognitive Disorders, 17, 269-273.

-Irish, M., Graham, A., Graham, K.S., Hodges, J.R. \& Hornberger, M. (2012). Differential impairment of source memory in progressive versus non-progressive behavioral variant frontotemporal dementia. Archives of Clinical Neuropsychology, 27, 338-347.

Jeong, Y., Cho, S.S., Park, J.M., Kang, S.J., Lee, J.S., Kang, E., ... Kim, S.E. (2005), 18F-FDG PET findings in frontotemporal dementia: An SPM analysis of 29 patients. Fournal of Nuclear Medicine, 46, 233-239.

Kanda, T., Ishii, K., Uemura, T., Miyamoto, N., Yoshikawa, T., Kono, A.K. \& Mori, E. (2008). Comparison of grey matter and metabolic reductions in frontotemporal dementia using FDG-PET and voxel-based morphometric MR studies. European fournal of Nuclear Medicine and Molecular Imaging, 35, 2227-2234.

Kerchner, G.A., Tartaglia, M.C. \& Boxer, A. (2011). Abhorring the vacuum: Use of Alzheimer's disease medications in frontotemporal dementia. Expert Review of Neurotherapeutics, 11, 709-717.

Kertesz, A. (2008). Frontotemporal dementia: A topical review. Cognitive and Behavioral Neurology, 21, 127-133.

-Kipps, C.M., Davies, R.R., Mitchell, J., Kril, J.J., Halliday, G.M. \& Hodges, J.R. (2007). Clinical significance of lobar atrophy in frontotemporal dementia: Application of an MRI visual rating scale. Dementia and Geriatric Cognitive Disorders, 23, 334-342.

Kitabayashi, Y., Otakara, C., Hirosawa, R., Narumoto, J. \& Fukui, K. (2005). Frontotemporal dementia complicated with schizophrenia. Psychiatry and Clinical Neurosciences, 59, 749-750.

Kleist, K. (1960). Schizophrenic symptoms and cerebral pathology. Fournal of Mental Science, 106, 246-255.

-Kosmidis, M.H., Aretouli, E., Bozikas, V.P., Giannakou, M. \& Ioannidis, P. (2008). Studying social cognition in patients with schizophrenia and patients with frontotemporal dementia: Theory of mind and the perception of sarcasm. Behavioral Neurology, 19, 65-69.

Kraepelin, E. (1919). Dementia Praecox and Paraphrenia. Edinburgh: Livingstone.

Lagodka, A. \& Robert, P. (2009). La schizophrénie tardive est-elle secondaire à des processus neurodégénératifs? Une revue de la littérature [Is late-onset schizophrenia related to neurodegenerative processes? A review of literature]. Encephale, 35, 386393.

Lawrie, S.M. \& Abukmeil, S.S. (1998). Brain abnormality in schizophrenia. A systematic and quantitative review of volumetric magnetic resonance imaging studies. British fournal of Psychiatry, 172, 110-120.

Le Ber, I., Camuzat, A., Hannequin, D., Pasquier, F., Guedj, E., Rovelet-Lecrux, A., ... French Research Network on FTD/ FTD-MND. (2008). Phenotype variability in progranulin mutation carriers: A clinical, neuropsychological, imaging and genetic study. Brain, 131, 732-746.

-Le Ber, I., Guedj, E., Gabelle, A., Verpillat, P., Volteau, M., Thomas-Anterion, C., ... Dubois, B. (2006). Demographic, neurological and behavioural characteristics and brain perfusion SPECT in frontal variant of frontotemporal dementia. Brain, 129, 3051-3065.

-Levin, S. (1984). Frontal lobe dysfunctions in schizophrenia - II. Impairments of psychological and brain functions. Fournal of Psychiatric Research, 18, 57-72.

Liddle, P.F. (1987). Schizophrenic syndromes, cognitive performance and neurological dysfunction. Psychological Medicine, 17, 49-57.

Lindau, M., Almkvist, O., Kushi, J., Boone, K., Johansson, S.E., Wahlund, L.O., ... Miller, B.L. (2000). First symptoms - Frontotemporal dementia versus Alzheimer's disease. Dementia and Geriatric Cognitive Disorders, 11, 286-293.
Luauté, J.P., Bidault, E. \& Sanabria, E. (1994). Atrophie corticale localisée temporale gauche et troubles schizophréniques : à propos de six cas [Cortical atrophy of the left temporal lobe and schizophrenia. Apropos of 6 cases]. Annales MedicoPsychologiques (Paris), 152, 246-251.

Luders, E., Narr, K.L., Thompson, P.M., Rex, D.E., Jancke, L. \& Toga, A.W. (2006). Hemispheric asymmetries in cortical thickness. Cerebral Cortex, 16, 1232-1238.

McKhann, G.M., Albert, M.S., Grossman, M., Miller, B.L., Dickson, D., Trojanowski, J.Q. \& Work Group on Frontotemporal Dementia and Pick's Disease (2001). Clinical and pathological diagnosis of frontotemporal dementia: Report of the Work Group on Frontotemporal Dementia and Pick's Disease. Archives of Neurology, 58, 1803-1809.

Mendez, M.F., Shapira, J.S., Woods, R.J., Licht, E.A. \& Saul, R.E. (2008). Psychotic symptoms in frontotemporal dementia: Prevalence and review. Dementia and Geriatric Cognitive Disorders, 25, 206-211.

-Mesulam, M.M. (2003). Primary progressive aphasia - A language-based dementia. New England fournal of Medicine, 349, 1535-1542.

Mesulam, M., Wicklund, A., Johnson, N., Rogalski, E., Leger, G.C., Rademaker, A., ... Bigio, E.H. (2008). Alzheimer and frontotemporal pathology in subsets of primary progressive aphasia. Annals of Neurology, 63, 709-719.

Momeni, P., DeTucci, K., Straub, R.E., Weinberger, D.R., Davies, P., Grafman, J., ... Huey, E.D. (2010b). Progranulin (GRN) in two siblings of a Latino family and in other patients with schizophrenia. Neurocase, 16, 273-279.

-Momeni, P., Wickremaratchi, M.M., Bell, J., Arnold, R., Beer, R., Hardy, J., ... Morris, HR. (2010a). Familial early onset frontotemporal dementia caused by a novel S356T MAPT mutation, initially diagnosed as schizophrenia. Clinical Neurology and Neurosurgery, 112, 917-920.

Murray, M.E., DeJesus-Hernandez, M., Rutherford, N.J., Baker, M., Duara, R., Graff-Radford, N., ... Dickson, D.W. (2011). Clinical and neuropathological heterogeneity of c9FTD/ALS associated with hexanucleotide repeat expansion in C9ORF72. Acta Neuropathologica, 122, 673-690.

- Neary, D., Snowden, J.S, Gustafson, L., Passant, U., Stuss, D., Black, S., ... Benson, D.F. (1998). Frontotemporal lobar degeneration: A consensus on clinical diagnostic criteria. Neurology, 51, 1546-1554.

Pantelis, C., Barnes, T.R. \& Nelson, H.E. (1992). Is the concept of frontal-subcortical dementia relevant to schizophrenia? British Fournal of Psychiatry, 160, 442-460.

Pennington, C., Hodges, J.R. \& Hornberger, M. (2011). Neural correlates of episodic memory in behavioral variant frontotemporal dementia. Fournal of Alzheimer's Disease, 24, 261-268.

Pick, A. (1892). Uber die Beziehung der senilen Hirnatrophie zur Aphasie [Relationship between senile brain atrophy and aphasia]. Pragischer Medizinischer Wochenschrift, 17, 165-167.

Piguet, O., Brooks, W.S., Halliday, G.M., Schofield, P.R., Stanford, P.M., Kwok, J.B., ... Hodges, J.R. (2004). Similar early clinical presentations in familial and non-familial frontotemporal dementia. Fournal of Neurology, Neurosurgery, and Psychiatry, 75, 1743-1745.

Puri, B.K., Hall, A.D. \& Lewis, S.W. (1994) Cerebral hemiatrophy and schizophrenia. British fournal of Psychiatry, 165, 403-405.

Rabinovici, G.D., Jagust, W.J., Furst, A.J., Ogar, J.M., Racine, C.A., Mormino, E.C., ... Gorno-Tempini, M.L. (2008). Abeta amyloid and glucose metabolism in three variants of primary progressive aphasia. Annals of Neurology, 64, 388-401.

Rademakers, R. (2012). C9orf72 repeat expansions in patients with ALS and FTD. Lancet Neurology, 11, 297-298.

-Rascovsky, K., Hodges, J.R., Knopman, D., Mendez, M.F., Kramer, J.H., Neuhaus, J., van Swieten, J.C., ... Miller, B.L. (2011). Sensitivity of revised diagnostic criteria for the 
behavioural variant of frontotemporal dementia. Brain, 134, 2456-2477.

Reischle, E., Sturm, K., Schuierer, G. \& Ibach, B. (2003). Ein Fall von schizophrenieformer Störung bei frontotemporaler Demenz (FTD) [Frontotemporal dementia presenting as acute late onset schizophrenia]. Psychiatrische Praxis, 30, S78-82.

Renton, A.E., Majounie, E., Waite, A., Simon-Sanchez, J., Rollinson, S., Gibbs, J.R., ... Traynor, B.J. (2011). A hexanucleotide repeat expansion in C9ORF72 is the cause of chromosome 9p21-linked ALS-FTD. Neuron, 72, 257-268.

Reynolds, J. (1858). On the pathology of convulsions, with special reference to those of children. Liverpool Medico-Chirurgie fournal, 2, 1-14.

Rohrer, J.D. \& Warren, J.D. (2011). Phenotypic signatures of genetic frontotemporal dementia. Current Opinion in Neurology, 24, 542-549.

Rohrer, J.D., Clarkson, M.J., Kittus, R., Rossor, M.N., Ourselin, S., Warren, J.D. \& Fox, N.C. (2012). Rates of hemispheric and lobar atrophy in the language variants of frontotemporal lobar degeneration. Fournal of Alzheimer's Disease, 30, 407-411.

Rohrer, J.D., Lashley, T., Schott, J.M., Warren, J.E., Mead, S., Isaacs, A.M., ... Warren, J.D. (2011) Clinical and neuroanatomical signatures of tissue pathology in frontotemporal lobar degeneration. Brain, 134, 2565-2581.

Rosenberg, R.N. (1983). Clinical Neurosciences: Neuropathology. New York: Churchill Livingstone.

-Schobel, S.A., Kelly, M.A., Corcoran, C.M., Van Heertum, K., Seckinger, R., Goetz, R., ... Malaspina, D. (2009). Anterior hippocampal and orbitofrontal cortical structural brain abnormalities in association with cognitive deficits in schizophrenia. Schizophrenia Research, 114, 110-118.

-Schoder, D., Hannequin, D., Martinaud, O., Opolczynski, G., Guyant-Maréchal, L., Le Ber, I. \& Campion, D. (2010). Morbid risk for schizophrenia in first-degree relatives of people with frontotemporal dementia. British fournal of Psychiatry, 197, 28-35.

-Seelaar, H., Klijnsma, K.Y., de Koning, I., van der Lugt, A., Zheng Chiu, W., Azmani, A., ... van Swieten, J.C. (2010). Frequency of ubiquitin and FUS-positive, TDP-43-negative frontotemporal lobar degeneration. Fournal of Neurology, 257, 747-753.

-Seelaar, H., Rohrer, J.D., Pijnenburg, Y.A., Fox, N.C., \& van Swieten, J.C. (2011). Clinical, genetic and pathological heterogeneity of frontotemporal dementia: A review. Fournal of Neurology, Neurosurgery, and Psychiatry, 82, 476-486.

-Seeley, W.W., Bauer, A.M., Miller, B.L., Gorno-Tempini, M.L., Kramer, J.H., Weiner, M. \& Rosen, H.J. (2005). The natural history of temporal variant frontotemporal dementia. Neurology, 64, 1384-1390.

-Shenton, M.E., Dickey, C.C., Frumin, M. \& McCarley, R.W. (2001). A review of MRI findings in schizophrenia. Schizophrenia Research, 49, 1-52.

-Snowden, J.S., Hu, Q., Rollinson, S., Halliwell, N., Robinson, A., Davidson, Y.S., ... Mann, D.M. (2011). The most common type of FTLD-FUS (aFTLD-U) is associated with a distinct clinical form of frontotemporal dementia but is not related to mutations in the FUS gene. Acta Neuropathologica, 122, 99-110.

-Snowden, J.S., Rollinson, S., Thompson, J.C., Harris, J.M., Stopford, C.L., Richardson, A.M., ... Pickering-Brown, S.M. (2012). Distinct clinical and pathological characteristics of frontotemporal dementia associated with C9ORF72 mutations. Brain, 135, 693-708.

-Spironelli, C., Angrilli, A., Calogero, A. \& Stegagno, L. (2011). Delta EEG band as a marker of left hypofrontality for language in schizophrenia patients. Schizophrenia Bulletin, 37, $757-767$.
Sumich, A., Chitnis, X.A., Fannon, D.G., O'Ceallaigh, S., Doku, V.C., Falrowicz, A., ... Sharma, T. (2002). Temporal lobe abnormalities in first-episode psychosis. American fournal of Psychiatry, 159, 1232-1235.

Sun, T. \& Walsh, C.A. (2006). Molecular approaches to brain asymmetry and handedness. Nature Reviezws Neuroscience, 7, 655-662.

Sun, T., Patoine, C., Abu-Khalil, A., Visvader, J., Sum, E., Cherry, T.J. \& ... Walsh, C.A. (2005). Early asymmetry of gene transcription in embryonic human left and right cerebral cortex. Science, 308, 1794-1798.

- van den Heuvel, M.P., Mandl, R.C., Stam, C.J., Kahn, R.S. \& Hulshoff, H.E. (2010). Aberrant frontal and temporal complex network structure in schizophrenia: A graph theoretical analysis. Fournal of Neuroscience, 30, 15915-15926.

Vanderzeypen, F., Bier, J.C., Genevrois, C., Mendlewicz, J. \& Lotstra, F. (2003). Frontal dementia or dementia praecox? A case report of a psychotic disorder with a severe decline. Encephale, 29, 172-180.

van Haren, N.E., Schnack, H.G., Cahn, W., van den Heuvel, M.P., Lepage, C., Collins, L., ... Kahn RS. (2011). Changes in cortical thickness during the course of illness in schizophrenia. Archives of General Psychiatry, 68, 871-880.

-Velakoulis, M., Walterfang, M., Mocellin, R., Pantelis, C. \& McLean, C. (2009). Frontotemporal dementia presenting as schizophrenia-like psychosis in young people: Clinicopathological series and review of cases. British Fournal of Psychiatry, 194, 298-305.

Waddington, J.L., Youssef, H. A., Farrell, M.A. \& Toland, J. (1995) Initial 'schizophrenia-like' psychosis in Pick's disease: Case study with neuroimaging and neuropathology, and implications for frontotemporal dysfunction in schizophrenia. Schizophrenia Research, 18, 79-82.

Weickert, T.W., Leslie, F., Rushby, J.A., Hodges, J.R. \& Hornberger, M. (2011). Probabilistic association learning in frontotemporal dementia and schizophrenia. Cortex. doi:10.1016/ j.cortex.2011.09.011

Weinberger, D.R. (1988). Schizophrenia and the frontal lobe. Trends in Neurosciences, 11, 367-370.

-Weinberger, D.R. \& Berman, K.F. (1988). Speculation on the meaning of cerebral metabolic hypofrontality in schizophrenia. Schizophrenia Bulletin, 14, 157-168.

Whitwell, J.L., Sampson, E.L., Watt, H.C., Harvey, R.J., Rossor, M.N. \& Fox, N.C. (2005). A volumetric magnetic resonance imaging study of the amygdala in frontotemporal lobar degeneration and Alzheimer's disease. Dementia and Geriatric Cognitive Disorders, 20, 238-244.

Whitwell, J.L., Xu, J., Mandrekar, J., Boeve, B.F., Knopman, D.S., Parisi, J.E., ... Josephs, K.A. (2012). Frontal asymmetry in behavioral variant frontotemporal dementia: Clinicoimaging and pathogenetic correlates. Neurobiology and Aging. doi:10.1016/ j.neurobiolaging.2012.03.009

Winograd-Gurvich, C., Fitzgerald, P.B., Georgiou-Karistianis, N., Bradshaw, J.L. \& White, O.B. (2006). Negative symptoms: A review of schizophrenia, melancholic depression and Parkinson's disease. Brain Research Bulletin, 70, 312-321.

-Wolf, R.C., Höse, A., Frasch, K., Walter, H. \& Vasic, N. (2008). Volumetric abnormalities associated with cognitive deficits in patients with schizophrenia. European Psychiatry, 23, 541-548.

Ziauddeen, H., Dibben, C., Kipps, C., Hodges, J.R. \& McKenna, P.J. (2011). Negative schizophrenic symptoms and the frontal lobe syndrome: One and the same? European Archives of Psychiatry and Clinical Neuroscience, 261, 59-67. 